\title{
Topical capsaicin-a novel and effective treatment for idiopathic intractable pruritus ani: a randomised, placebo controlled, crossover study
}

\author{
J Lysy, M Sistiery-Ittah, Y Israelit, A Shmueli, N Strauss-Liviatan, V Mindrul, D Keret, \\ E Goldin
}

Gut 2003;52:1323-1326

See end of article for authors' affiliations

Correspondence to:

Dr E Goldin, Department of

Gastroenterology,

Hadassah University

Hospital, Ein Kerem, PO

Box 12000, Jerusalem

91120 , Israel

goldin@cc.huji.ac.il

Accepted for publication 8 January 2003
Purpose: Pruritus ani is a common and embarrassing proctological condition which can be very difficult to treat. We report the results of a double blind placebo controlled study of treatment with capsaicin.

Methods: Firstly, a pilot open study was carried out on five patients to establish which of two doses was the most acceptable by comparing effectiveness and side effects. Secondly, a double blind, placebo controlled, crossover study of topical capsaicin was performed. This study involved two four week treatment phases separated by a one week washout phase. Forty four patients were randomised to receive locally either active capsaicin $(0.006 \%$ ) or placebo (menthol $1 \%$ ) ointment over a four week period (22 patients per group). After four weeks of treatment and a one week washout period, the placebo group began to receive capsaicin while the treated group received placebo (menthol 1\%) for another four weeks. At the end of the controlled study, responders from both groups continued with capsaicin treatment in an open labelled manner.

Results: Thirty one of 44 patients experienced relief during capsaicin treatment periods and did not respond to menthol; all patients not responding to capsaicin also failed on menthol ( $p<0.0001)$. In 13 patients, treatment with capsaicin was unsuccessful: eight patients did not respond to capsaicin treatment, one responded equally to capsaicin and placebo, and four others dropped out because of side effects. During the follow up period (mean 10.9 (SD 5.8) months), 29 "responders" needed a mean application of capsaicin every day (1.6 (SD 1.2); range 0.5-7 days) to remain symptom free (or nearly symptom free).

Conclusion: Capsaicin is a new, safe, and highly effective treatment for severe intractable idiopathic pruritus ani.
$\mathrm{P}$ uritus ani is a common proctological problem, characterised by intense itching localised in the anus and perianal skin. Pruritus ani may result from an underlying disorder of the epithelium in that area or from anorectal pathology. Many patients with pruritus ani do not have a clear cause for the condition. Faecal contamination of the perineum in the absence of gross soiling, irritant chemicals in faeces, allergies to locally applied agents or components of diet, and even psychosomatic factors have been suggested as possible aetiologies but are not conclusively proved to be of relevance. ${ }^{1}$ When pruritus ani has no demonstrable aetiology, it is often described as idiopathic, and advice regarding hygiene and drying methods is usually given, with poor results. ${ }^{2}$ Capsaicin is a natural alkaloid derived from plants of the Solanaceae family. Topical capsaicin is known to be effective and safe in the treatment of pain and itching. ${ }^{3}$ Although the precise mechanism of action is not fully understood, evidence suggests that capsaicin exercises an active depressant effect in the synthesis, storage, transport, and release of substance $\mathrm{P}^{4}$ Substance $\mathrm{P}$ is thought to be an important neuropeptide that acts as a mediator of pain and itching impulses from the periphery to the central nervous system. ${ }^{56}$ Capsaicin probably elicits its effect on $\mathrm{C}$ fibre sensory afferent neurones which contains substance $\mathrm{P}$, by activating capsaicin (vanilloid) receptor. The capsaicin (vanilloid) receptor is a ligand gated non-selective cation channel expressed predominantly by sensory neurones. Capsaicin functions to activate and then, at higher doses and longer times, desensitise this class of neurones. ${ }^{7}$ We report for the first time the results of a double blind placebo controlled study of capsaicin treatment in chronic idiopathic and intractable pruritus ani.

\section{METHODS}

\section{Study sample}

Consecutive patients with chronic pruritus ani who attended the proctological outpatients clinic at the hospital and an affiliated ambulatory clinic were recruited. Forty nine patients with chronic idiopathic anal itching of at least three months' duration were enrolled. Patients with obvious pathological lesions, such as dermatological diseases, faecal incontinence, previous anal surgery, or significant haemorrhoids, were excluded. None of the patients had responded to traditional treatment which included gentle hygiene, drying methods, avoidance of soap, and avoidance of coffee or other dietary items that seemed to exacerbate symptoms. A topical steroid was prescribed to patients with dermatosis.

\section{Study design}

All patients underwent a pretreatment evaluation. Their history concerning duration and severity of symptoms, use of other topical agents, associated medical illness, and daily coffee consumption were recorded. Clinical investigation included full perianal skin examination, skin scraping for fungal detection, digital rectal examination, anoscopy, and sigmoidoscopy or colonoscopy. Fourteen patients in whom the possibility of rectal dysmotility or sphincter damage was raised also had anorectal manometry and anal endosonography. 
Table 1 Patient characteristics after randomisation (study 2)

\begin{tabular}{lll}
\hline & $\begin{array}{l}\text { Capsaicin (first) } \\
\text { group A }\end{array}$ & $\begin{array}{l}\text { Menthol (first) } \\
\text { group B }\end{array}$ \\
\hline No of patients & 22 & 22 \\
Age (y)* & $50.9(13.7)$ & 49.9 (13.9) \\
Sex ratio (M:F) & $9: 13$ & $11: 11$ \\
Duration of symptoms & $24.7(21.2)$ & 23.1 (19.1) \\
(months)* & & \\
\hline *Values are mean (SD). & & \\
\end{tabular}

\section{Study 1}

An open pilot investigation to establish which of two doses was the most acceptable by comparing effectiveness and side effects was carried out in five patients. Firstly, these patients received a topical capsaicin preparation $(0.012 \%)$, three times a day for two weeks, and later a $0.006 \%$ capsaicin dose for another two weeks. Comparison of itching and side effects was recorded.

\section{Study 2}

This was a double blind, random order, placebo controlled, crossover study involving two four week treatment phases, separated by a one week washout phase. Forty four patients with intractable idiopathic pruritus ani were randomised to receive a very thin layer of perianal topical treatment (group A: 22 patients, capsaicin cream $0.006 \%$ ) or control treatment (group B: 22 patients, menthol 1\%), three times a day for four weeks. A one week washout period was then allowed and after that patients from group A received menthol ( $1 \%)$ while group $B$ patients received capsaicin for four weeks under the same conditions and doses described above. Randomisation was done in the hospital pharmacy using a table of random numbers. Trial ointments were prepared by staff not involved further in the trial. Both trial ointments looked the same. The randomisation code remained in the hospital pharmacy during the trial and was not available to the investigators. The code was broken at the ninth week or earlier when significant side effects prevented study continuation.

Patient characteristics are illustrated in table 1. Patients were of similar age and sex, and duration of symptom were similar in both groups.

At the end of the controlled phase of the study, responders from both groups were asked to use capsaicin only if itching recurred.

\section{Materials}

The ointment used for this study was prepared in the hospital pharmacy from capsaicin $0.025 \%$ (Zostrix $0.025 \%$; Rafa Laboratories, Israel), diluted $1: 2$ and $1: 4$ in white soft paraffin to give a final capsaicin concentration of $0.012 \%$ and $0.006 \%$. Menthol cream (1\%) was also prepared in the hospital pharmacy. Placebo and active cream were supplied in identical containers.

Patients kept a 28 day symptom diary during both of the four week treatment phases. This diary recorded the daily occurrence of itching, perianal burning sensation following cream application, duration of symptoms, and other side effects. Itching and burning were each scored 1-5 ( 1 no symptoms, 5 maximum symptoms). The degree of itching before treatment was scored as 5 for each patient. Itching scores grade 1 (no symptoms) and grade 2 (nearly complete symptomatic relief with sporadic episodes of mild itching) following capsaicin or menthol application were defined as positive responses $(\mathrm{Y})$. Itching scores grade 3-5 following capsaicin or menthol application were defined as negative responses $(\mathrm{N})$. At the end of nine weeks, responders from both groups who wished to continue with capsaicin treatment were provided with capsaicin and were asked to record on a diary, once weekly, the frequency and degree of itching as well as the degree of post application burning sensation. The ethics committee of the hospital approved the study, and written informed consent was obtained from each participant at the beginning of the study.

\section{Follow up}

Patients were advised to continue hygienic and drying methods. No patient was treated with topical steroids or anaesthetics during the study. All patients received clinical examinations and were asked about side effects and whether they wanted to continue to participate in the study. In the pilot group, evaluation was performed almost every day, while in the controlled study, at four and nine weeks. At the end of the controlled study, patients were followed every 2-3 months when they came again to receive capsaicin.

\section{Statistical analysis}

The outcome of each group was evaluated clinically. Response was defined as complete symptomatic relief (grade 1) or nearly complete symptomatic relief with sporadic episodes of mild itching (grade 2).

Analysis was based on differences for matched pairs of values measured in patients: we used the Wilcoxon signed ranks test on matched pairs to compare scores for side effects and duration of capsaicin versus menthol treatments and, in a similar way, to compare scores for responses to capsaicin at the beginning of the trial versus follow up, and of side effects at the beginning versus the end of the trial.

To compare responses ( $\mathrm{Y} / \mathrm{N}$ ) to capsaicin versus menthol, we used the McNemar $\chi^{2}$ test for the significance of changes.

Comparison of all background as well as researched variables over the two treatments in our cross designed trial was performed using the $\chi^{2}$ test and two sample $t$ test, as appropriate.

As the washout period lasted one week and as the beneficial effect of capsaicin disappears after 1-5 days (see pilot study), we tended to assume that there were no carryover effects. Our assumption was verified using a model of GLM with repeated measurements to test for carryover effects in the response and side effects variables, where none was found to be significant.

\section{RESULTS}

\section{Clinical investigation}

Anal manometry revealed low-normal resting pressure in two patients $(40 \mathrm{~mm} \mathrm{Hg})$ and decreased maximum tolerated volume in three patients. Anal endosonography revealed a thinned $(1.5 \mathrm{~mm})$ internal anal sphincter in one of the patients with low-normal resting pressure. Evidence of mild faecal soiling was found in five patients.

\section{Study No 1: dose determination}

At a concentration of $0.012 \%$, capsaicin caused perianal burning immediately following application. Although this lasted only 30-60 minutes, three of five patients had to discontinue this preparation after two weeks of treatment. During this treatment period, itching disappeared in all patients. Application of the lower concentration of capsaicin $(0.006 \%)$ was followed by a very mild perianal burning lasting 10-15 minutes in four patients. Only one patient treated with this preparation had moderate burning and dropped out of the study. All other patients completed the two weeks of treatment with this concentration. In four patients, itching had completely resolved. At four weeks, patients were advised to apply capsaicin only when itching recurred. During a mean follow up of 18 months (range 16-22), these patients required a single daily application of capsaicin in a mean time interval of 2.5 days (range 2-4). Apart from the above mentioned mild perianal burning, no other side effects were described. 


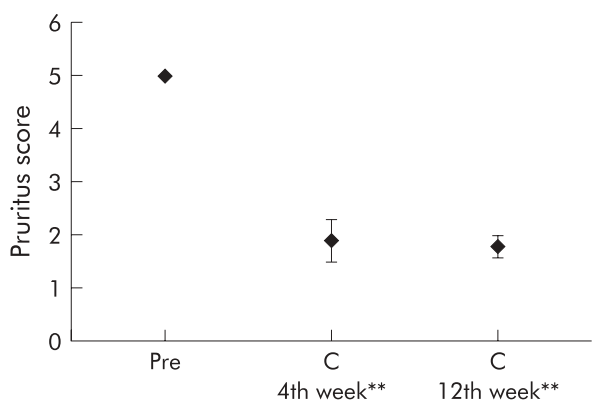

Figure 1 Treatment efficacy. Pruritus score at week 4 and week 12 following application of capsaicin (C). ${ }^{*} p<0.0001$ compared with pretreatment (Pre) values.

\section{Study No 2: controlled study}

Response $(\mathrm{Y} / \mathrm{N})$ to capsaicin was significantly better than to menthol $(p<0.0001$ by McNemar test): 31 of 44 patients experienced relief during capsaicin treatment periods and did not respond to menthol; all patients not responding to capsaicin also failed on menthol. In responders, the itching score decreased significantly under capsaicin treatment compared with pretreatment values and was unchanged during the long term follow up period $(\mathrm{p}<0.001)$ (fig 1 , table 2$)$. The beneficial effect of capsaicin was immediate in 24 patients or within three days in seven patients. Four patients dropped out because of side effects-three patients because of capsaicin induced moderate to severe perianal burning and one patient developed urticaria while treated with capsaicin. Eight patients dropped out due to treatment failure, and in one patient symptoms disappeared after both capsaicin and menthol application. All patients had some degree of perianal burning following each capsaicin or menthol application. The burning sensation was usually mild following both application preparations, but burning score and duration were significantly higher and longer following capsaicin application $(\mathrm{p}<0.001)$ (table 3$)$. The burning sensation following capsaicin application decreased significantly after four weeks of application $(\mathrm{p}<0.001)$ but did not disappear completely. Two patients suffered from moderate burning following capsaicin application but wished to continue the study and use capsaicin after the end of the controlled trial.

\section{Long term follow up}

At the end of the controlled trial, patients from both groups who responded to capsaicin treatment were advised to continue with capsaicin application only when itching recurred. During the follow up period (mean 10.9 (SD 5.8) months), 29 "responders" needed a mean application of capsaicin every day (1.6 (SD 1.2); range $0.5-7$ days) to remain symptom free (or nearly symptom free).

Eighteen patients were followed for more than eight months (mean 14.9 (SD 3.3); range 10-20 months). All patients remained asymptomatic (four patients) or nearly asymptomatic (14 patients) and required single daily capsaicin application at a mean interval time of 1.97 (SD 1.4) days (range 0.5-7). During this time, no side effects other than very mild perianal burning were reported. Shorter term follow up patients ( 11 patients, mean 4.9 (SD 2.2); range 2-8 months) required capsaicin at a mean time interval of 0.96 days (SD 0.4 ; range $0.5-2$ ) to become symptom free (three patients) or nearly symptom free (eight patients). The difference was significant $(p=0.025)$ between short and long term follow up patients only for capsaicin mean time interval application (mean 0.958 (SD 1.4) $v$ mean 1.972 (SD 0.4)).

Two patients gradually increased the application frequency during the follow up period from one application every three days to daily application. Two patients experienced a decrease in capsaicin effect but this was restored following capsaicin application $(0.012 \%)$.

\section{DISCUSSION}

Itching of the anus is a common condition afflicting up to 5\% of the population. ${ }^{2}$ The incidence of primary and secondary pruritus ani varies among different studies. Several studies show that the incidence of idiopathic pruritus ani is $75-95 \% .{ }^{9}$ Other authors have found that its incidence is only $25 \% .{ }^{10}$

\begin{tabular}{|c|c|c|c|c|c|c|c|}
\hline & \multicolumn{2}{|c|}{ Capsaicin (C) } & \multicolumn{2}{|c|}{ Menthol (M) } & \multicolumn{2}{|l|}{$C-M$} & \multirow[b]{2}{*}{$\mathrm{p}$ Value } \\
\hline & Mean & SD & Mean & SD & Mean & SD & \\
\hline $\begin{array}{l}\text { Therapeutic effect score at } \\
4 \text { weeks }\end{array}$ & 1.87 & 1.19 & 4.42 & 0.64 & -2.55 & 1.31 & $<0.001$ \\
\hline $\begin{array}{l}\text { Burning sensation score } \\
\text { (side effect) }\end{array}$ & 2.60 & 0.70 & 2.24 & 0.43 & 0.36 & 0.53 & $<0.001$ \\
\hline Side effect duration (min) & 14.64 & 5.56 & 12.02 & 3.67 & 2.62 & 4.17 & $<0.001$ \\
\hline
\end{tabular}

\begin{tabular}{|c|c|c|c|c|c|}
\hline Outcome measure & Group & Capsaicin & Menthol & $\begin{array}{l}\text { Treatment effect } \\
\text { (p value) }\end{array}$ & $\begin{array}{l}\text { Carryover effect } \\
\text { (p value) }\end{array}$ \\
\hline \multirow[t]{3}{*}{ Therapeutic effect } & $A+B$ & $1.90(1.19)$ & $4.43(0.64)$ & $<0.001$ & 0.214 \\
\hline & A & $1.80(1.20)$ & $4.65(0.49)$ & & \\
\hline & B & $2.00(1.20)$ & $4.20(0.70)$ & & \\
\hline \multirow[t]{3}{*}{ Burning sensation } & $A+B$ & $2.65(0.78)$ & $2.24(0.43)$ & $<0.001$ & 0.935 \\
\hline & A & $2.71(0.78)$ & $2.25(0.44)$ & & \\
\hline & B & $2.59(0.80)$ & $2.23(0.43)$ & & \\
\hline \multirow[t]{3}{*}{ Side effect duration } & $A+B$ & $14.64(5.56)$ & $12.02(3.67)$ & $<0.001$ & 0.201 \\
\hline & A & $14.25(4.38)$ & $12.50(4.73)$ & & \\
\hline & $B$ & $15 \quad(6.55)$ & $11.59(2.38)$ & & \\
\hline
\end{tabular}


Characteristically, pruritus ani is exacerbated by friction or a warm moist perineal environment. Poor anal hygiene or, in contrast, overcleansing with soap is often a contributing factor. Patients with idiopathic pruritus ani have been found to have abnormal transient internal sphincter relaxation ${ }^{11}$ and an abnormally profound decrease in anal canal pressure during rectal distention, as well as early incontinence on saline continence tests, implying that intermittent seepage from the anal canal may be a causative factor. ${ }^{12}$ A decrease in resting anal canal pressure with coffee intake has also been reported ${ }^{13}$ which may explain the documented exacerbation of symptoms with ingestion of caffeine. Treatment of idiopathic pruritus ani is non-specific and is aimed at restoring clean dry intact skin. The perineum must be gently cleaned with water after defecation. Drying should also be done gently, preferably with a hair dryer. The perineum may be cleaned several times a day with water premoistened wipes to absorb seepage material. Patients should be advised on the use of emulsifying ointment as a soap substitute, and not to shampoo their hair in the bath. Avoidance of impervious underwear, such as acrylic and nylon garments which trap sweat, and use of a drying powder may be helpful. Short term use of topical steroids for up to one month is useful for patients with dermatosis. Some patients with idiopathic pruritus ani respond favourably to such conservative management. Unfortunately, a significant percentage remains very difficult to treat and represents an important clinical challenge. Attempts to treat refractory pruritus ani include perianal injection of anaesthetic agents, ${ }^{14}$ surgical disruption of the sensory nerve supply to the perineal area, ${ }^{15}$ cryotherapy, ${ }^{16}$ methylene blue injection, ${ }^{17}$ and even hypnotherapy. ${ }^{18}$ Most of these attempts have had limited success, and some have had significant side effects.

Capsaicin is a natural alkaloid extracted from red chili peppers. Its pharmacological action is mainly depletion of substance P from sensory neurones. Capsaicin binds specifically to type C sensory neurones, induces release of substance $\mathrm{P}$, followed by inhibition of synthesis, transport, and storage of this neuropeptide. ${ }^{5}$ Topical capsaicin has been described as a safe and effective medicine for the relief of pain associated with postherpetic neuralgia, rheumatoid arthritis, and several other pain related conditions. It has also been shown that capsaicin is effective in the treatment of histamine induced pruritus, aquagenic pruritus, itching associated with uraemia, nodular prurigo, and pruritus related to postmastectomy syndrome. ${ }^{3}$

In the present study, only patients with chronic (longer than three months' duration) intractable pruritus ani were included. Topical capsaicin cream in concentrations of $0.025 \%$, $0.5 \%$, and $1 \%$ had previously been used to treat itching in other pathologies. ${ }^{19}$ Although no serious side effects were reported, burning sensation at the site of application prevented its use in up to $30 \%$ of patients..$^{20}$ As the perianal region is a very sensitive area of the body, we first conducted a preliminary investigation to establish the acceptable drug concentration. In this study, we found that a concentration of $0.006 \%$ capsaicin was effective in alleviating pruritus ani without the significant burning sensation associated with more concentrated preparations.

Our study demonstrated, for the first time, that capsaicin can be used successfully to treat chronic intractable pruritus ani. Patients in this study experienced dramatic itching relief after the first day of treatment. Itching was relieved in 31 of 44 patients in the treated group compared with one in the placebo group $(\mathrm{p}<0.0001)$. Overall, five of 49 patients $(10 \%)$ dropped out of the study during the first week of capsaicin $0.006 \%$ application because of intolerable burning sensation (four patients) or urticaria (one patient). No other significant side effects or complications were noted. During the follow up period, the trial was conducted as an open label study and "responders" had stopped daily capsaicin applications. Although itching recurred sporadically in all patients, patients needed a mean application of capsaicin every 1.6 days (SD 1.2; range $0.5-7$ days) to remain symptom free (or near symptom free).

A long term follow up study showed that the therapeutic effect of capsaicin did not decrease in general over time. However, two patients needed more frequent capsaicin applications and two patients more concentrated capsaicin preparations in order to remain symptom free in the long term follow up groups. Burning sensation was mild in two patients receiving capsaicin $0.012 \%$. Burning sensation decreased in most patients over time and probably a more concentrated capsaicin preparation can be used in most patients, if required. Further studies are necessary to more precisely establish the range of effective capsaicin concentration for long term treatment. In the "responders" group, more patients were completely symptom free than nearly symptom free during the controlled phase compared with the follow up period. This was mainly due to the instructions given to the patients during the follow up period to apply capsaicin if symptoms recurred. Most of the patients who ignored this suggestion and applied capsaicin on a daily basis remained completely symptom free. Thus it is suggested to offer patients both possibilities and let them chose according to their preference.

In summary, perianal application of $0.006 \%$ capsaicin cream is a new and promising approach to the treatment of chronic idiopathic intractable pruritus ani. This drug concentration was significantly effective and safe in this pathology. The study also suggests that the neuropeptide substance P may play a key role as a mediator of itching in chronic pruritus ani.

Authors' affiliations

J Lysy, M Sistiery-Ittah, Y Israelit, A Shmueli, N Strauss-Liviatan, V Mindrul, D Keret, E Goldin, Department of Gastroenterology, Hadassah University Hospital, Hebrew University Medical School, Jerusalem, Israel

\section{REFERENCES}

Jones DJ. ABC of colorectal diseases. Pruritus ani. BM 1992:305:575-7.

2 Mazier WP. Hemorrhoids fissures and pruritus ani. Surg Clin North Am 1994; 74: 1277-92

3 Hautkappe M, Roizen MF, Toledano A, et al. Review of the effectiveness of capsaicin for painful cutaneous disorders and neural dysfunction. Clin J Pain 1998;14:97-106.

4 Burks TF, Buck SH, Miller MS. Mechanisms of depletion of substance P by capsaicin. Fed Proc 1985;44:2531-4.

5 Greaves MW, Wall PD. Pathophysiology of itching. Lancet 1996;348:938-40

6 Rumsfield JA, West DP. Topical capsaicin in dermatologic and peripheral pain disorders. DICP 1991;25:381-7.

7 Caterina MJ, Schumacher MA, Tominaga M, et al. The capsaicin receptor: a heat- activated ion channel in the pain pathway. Nature 1997:23:389:783-4

8 Szallasi A. Vanilloid (capsaicin) receptors in health and disease. Am J Clin Pathol 2002;1 18:1 10-21.

9 Bernardi RS, Chen HP. Perianal extramammary Paget's disease. Surg Gynecol Obstet 1988;167:359

10 Wexner SD, Daily TH. Pruritus ani: diagnosis and management. Curr Concepts Skin Dis 1986:7:5-9.

11 Farouk R, Duthie GS, Pryde A, et al. Abnormal transient internal sphincter relaxation in idiopathic pruritus ani: physiologic evidence from ambulatory monitoring. Br J Surg 1994;81:603-6.

12 Allan A, Ambrose NS, Silverman S. Physiological study of pruritus ani. Br J Surg 1987;74:576-9.

13 Smith LE, Henrichs D, McCullah RD. Prospective studies on the etiology and treatment of pruritus ani. Dis Colon Rectum 1982;25:358-63.

14 Gabriel WB. Treatment of pruritus ani and anal fissure: the use of anaesthetic solutions in oil. BM 1929;I:1070-2.

15 Bacon HE. Anus Rectum and Sigmoid Colon: Diagnosis and Treatment, vol 1, 3rd edn. Philadelphia: JB Lippincott; 1949

16 Detrano SJ. Cryotherapy for chronic specific pruritus ani. J Dermatol Surg Oncol 1984;10: 483-4.

17 Farouk R, Lee PWR. Intradermal methylene blue injection for the treatment of intractable idiopathic pruritus ani. BM 1997;84:670.

18 Rucklidge JJ, Saunders D. Hypnosis in a case of long-standing idiopathic itch. Psychosm Med 1999;61:355-8.

19 Lotti T, Teofoli P, Tsampau D. Treatment of aquagenic pruritus with topical capsaicin cream. J Am Acad Dermatol 1994:30:232-5.

20 Yosipovitch G, David M. The diagnostic and therapeutic approach to generalized pruritus - perspective of internal medicine. Med Update 1998; 18:11-17. 